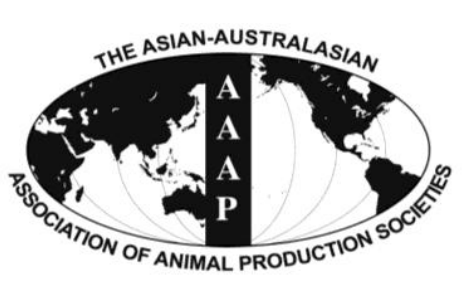

Open Access

\begin{tabular}{c} 
Open Access \\
Asian Australas. J. Anim. Sci. \\
Vol. 27, No. 4 : 561-566 April 2014 \\
http://dx.doi.org/10.5713/ajas.2013.13455 \\
\hline www.ajas.info \\
pISSN 101 1-2367 elSSN 1976-5517
\end{tabular}

\title{
Cloning and Sequence Analysis of Wild Argali ISG15 cDNA
}

\author{
Yanming Sun*, Kaili Chen', Wen Shen, Rupeng Cui, and Haifu Lu \\ College of Animal Science and Technology, Shihezi University, Shihezi, 832003, Xinjiang, China
}

\begin{abstract}
The complete coding sequence of Wild Argali ISG15 cDNA was generated by rapid amplification of cDNA ends. The ISG15 cDNA was $642 \mathrm{bp}$ with an open reading frame of $474 \mathrm{bp}$, which encoded a $17.47 \mathrm{kDa}$ protein composed of 157 amino acids. Its amino acid sequence shared 97.9\%, 80.8\%, 91.4\%, 94.3\%, 78.3\% identity with those of ISG15cDNA from Ovis aries (accession no. NM001009735.1), Capra hircus (accession no. HQ329186.1), Bos taurus (accession no. BC102318.1), Bubalus bubalis (accession no. HM543269.1), and Sus scrofa (accession no. EU647216.1), respectively. The entire coding sequence was inserted into the pET-28a vector and expressed in E. coli. The recombinant protein corresponded to the expected molecular mass of $25 \mathrm{kDa}$ as judged by SDSPAGE, and it was detected in the bacterial inclusion bodies. The expressed protein could be purified by $\mathrm{Ni}^{2+}$ chelate affinity chromatography and the results from the lymphocyte proliferation test showed that the product could stimulate lymphocyte proliferation very well ( $\mathrm{p}<0.05$ ), which further confirmed its biological activity. (Key Words: Argali, ISG15cDNA, Clone, Sequencing, Expression, Activity Detection)
\end{abstract}

\section{INTRODUCTION}

Argali (Ovis ammon), also known as the big sheep, Artiodactyla, Ruminantia, Boridae, Caprinae, genus Ovis, belongs to wild animals under the second class state protection (Yu et al., 2008). Wild Argali are distributed in the mountains of Eurasia, and also found in Inner Mongolia, Ningxia, Gansu, Xinjiang, Qinghai, and Tibet in China. Their main characteristics are: the rams weigh up to $200 \mathrm{~kg}$, both male and female have horns, the whole body is brown gray with a white hip spot, they have more lean and less fat content enabling fast growth, strong adaptability and high disease resistance as (Jueken et al., 2007).

One of the most abundantly expressed proteins in response to interferons (IFN) is IFN-stimulated gene product 15 (ISG15), which is not only seen during antiviral challenge but also during early pregnancy within the uterus of bovine, ovine, porcine, murine, human and baboon

\footnotetext{
* Corresponding Author: Sun Yanming. Tel: +86-0993-2027809, Fax: +86-0993-2058079, E-mail: sym@shzu.edu.cn

${ }^{1}$ College of Life Science, Shihezi University, Shihezi, 832003, Xinjiang, China.

Submitted Jul. 28, 2013; Accepted Nov. 27, 2013; Revised Dec. 23, 2013
}

species (Rempel et al., 2005). ISG15 is induced as a disease resistance gene and expressed efficiently in mammalian cells after stimuli with IFNs and viruses (Der et al., 1998). ISG15 is a $17.15 \mathrm{kDa}$ protein composed of 165 amino acid residues with further modifications after translation. ISG15 is related to disease resistance in sheep. As congenital immune response is the first line of defense against pathogens mainly through a series of specific cytokineinduced expression (Chang et al., 2006), the rapid high expression of ISG15 protein indicates that it may play an important role in innate immunity during infection.

However, there are no reports regarding the ISG15 sequence of Xinjiang Wild Argali. The encoded gene product corresponds to ubiquitin (Zhao et al., 2004), and the amino acids of ubiquitin are not conserved among different species (Zhang et al., 2011). To study the difference in nucleotide sequences and amino acid sequences of ISG15 gene between wild and domestic animals, the present study used the sequences from cattle and sheep as the references to clone and analyze the cDNA sequence by RACE technique and in vitro expression in prokaryotic cells, which provides a basis for further studies on the differences in gene functions and disease resistance. 


\section{MATEREALS AND METHODS}

\section{Peripheral blood materials and RNA preparation}

Heparinised blood $(2 \mathrm{mg} / \mathrm{mL})$ was collected by venipuncture from four healthy wild Argali. The peripheral blood lymphocyte cells (PBLCs) were separated by using the standard Ficoll-hypaque gradient centrifugation method (Haoyang Biological Manufacturer Co., LTD, Tianjin, China) according to the manufacturer's instructions. Total RNA was extracted by using the Trizol (Invitrogen, Beijing, China) method and stored at $-70^{\circ} \mathrm{C}$ until use.

\section{Rapid amplification of cDNA 5'ends (5'-RACE)}

The 5' ends were generated according to the instructions for the 5' Full RACE Core Set cDNA Kit (Clontech, Beijing, China). Briefly, cDNA was synthesized by reverse transcription (RT) UPM primer (as provided in the kit). Based on the published mRNA sequence of Ovis aries (accession no. NM001009735.1), Capra hircus (accession no. HQ329186.1) and Bos taurus (accession no. $\mathrm{BC} 102318.1)$, gene specific primers namely B1 (5'TTTGCTACAACTTTATTACTG3'), B2 (5'CTTTTCAA AAG-ACAGCCAGA3'), C1 (5'AAGCAGTGGTATCAAC GCAGAGTACGCGGGG3'), and C2 (5'-CCACTCAAA CTCCTGCACCAGCCTACC3') were employed in the nested PCR at concentration of $20 \mathrm{pmol} / \mu \mathrm{L}$ each. The second amplification products were recovered using 'Takara Agarose gel DNA purification Kit version 2.0' according to the manufacturer's instructions and ligated into pMD-18T cloning vector (Takara Biotechnology, Dalian, China) as described (Sambrook et al., 2002). Seven clones with inserts of the expected size were identified after EcoRI and HindIII digestion. The sequences in the seven clones were verified by the dideoxy-mediated DNA sequencing method using Taq DNA Polymerase (Qiagen, Shanghai, China).

\section{Rapid amplification of cDNA 3'ends (3'-RACE)}

The 3'ends were synthesized according to the instructions in 3'Full RACE Core Set cDNA Kit (Takara Biotechnology). The 3-sites adapter Primer (provided in the Kit), the D3 primer (5'GGGACC-TGAAGGTGAAG ATGCT3') and D4 primer (5'TGCCTCTGAGGGA CTCCATGAT3') were used at a concentration of 20 $\mathrm{pmol} / \mu \mathrm{L}$ each. The 3' end PCR products were purified and ligated into pMD-18T cloning vector (Takara Biotechnology). Six clones with inserts of the expected size were identified after EcoRI and HindIII digestion. The sequences in the six clones were verified by the dideoxymediated DNA sequencing method using Taq DNA Polymerase (Qiagen, Shanghai, China).

\section{Synthesis of complete ISG15 cDNA}

The complete coding sequence was generated by RT-
PCR as described (Sambrook et al., 2002). The primers used were as followed: P1 (5'GAATTCATGGGCG GGGACCTGA3'); P2 (5'AAGCTTTTTG-CTACAACTTT ATTCACTGCG3') at concentrations of $20 \mathrm{pmol} / \mu \mathrm{L}$ each. The purified PCR products were ligated into pMD-18T cloning vector (Takara Biotechnology, Dalian, China), transformed and sub-cultured. Ten randomly selected clones with inserts of the expected size were identified after EcoRI and HindIII digestion. The sequences of the ten clones were also verified by the dideoxy-mediated DNA sequencing method using Taq DNA Polymerase (Qiagen, Shanghai, China).

\section{Construction of expression vector}

The expression vector of recombinant ISG15 protein was constructed by inserting full length ISG15 cDNA into pET-28a plasmid (Takara Biotechnology, Dalian, China) as described (Sambrook et al., 2002). Briefly, recombinant pMD-18T with ISG15 cDNA was digested with EcoRI and HindIII and the ISG15 cDNA fragment was inserted into pET-28a vector previously linearized with similar enzymes to obtain the pET-28a/ISG15 cDNA expression vector. The recombinant vector was transformed into Escherichia coli and positive clones were verified by enzyme digestion.

\section{Expression of recombinant ISG15 protein}

Transformed Escherichia coli harboring pET-28a/ISG15 cDNA was sub-cultured in Lurria Bertani media supplemented with Kanamycin $(100 \mu \mathrm{g} / \mathrm{mL})$ and incubated at $37^{\circ} \mathrm{C}$ until the $\mathrm{OD}_{600}$ reached 0.4 to 0.6 . The expression was induced with isopropyI-B-D-thiogalactopyranoside (IPTG) (Sigma, Shanghai, China) at a final concentration of $1 \mathrm{mM}$. After $8 \mathrm{~h}$ of incubation at $37^{\circ} \mathrm{C}$, the cells were harvested and the expression level was assayed by SDSPAGE.

\section{Purification of recombinant ISG15 proteins}

Following induction, bacterial pellets were collected and recombinant proteins were isolated under denatured conditions as described by the manufacturer (Qiagen, Shanghai, China). Briefly, the bacterial pellet was mixed in $8 \mathrm{M}$ urea, $0.1 \mathrm{M} \mathrm{Na}_{2} \mathrm{HPO}_{4}, 0.01 \mathrm{M}$ Tris- $\mathrm{HCl}(\mathrm{pH} 8.0), 1 \mathrm{M}$ 2-mercaptoethanol, sonicated and centrifuged. The supernatant was applied to Ni-NTA agarose. The NTA column was washed with 20 volumes of $8 \mathrm{M}$ urea, $0.1 \mathrm{M}$ $\mathrm{Na}_{2} \mathrm{HPO}_{4}$ and $10 \mathrm{mM}$ Tris- $\mathrm{HCl}(\mathrm{pH}$ 6.3), followed by a 20 column volume of wash step with $8 \mathrm{M}$ urea and $50 \mathrm{mM}$ Tris $(\mathrm{pH}$ 8.0). Recombinant ISG15 proteins were refolded in 0.1 M urea, $50 \mathrm{mM}$ Tris-HCl, $2 \mathrm{M}$ oxidized glutathione (Sigma, Shanghai, China), $0.02 \mathrm{M}$ reduced glutathione (Sigma, Shanghai, China) $\mathrm{pH}$ 8.0. Protein renaturation was carried out on the Ni-NTA column using a linear gradient over a period of $2 \mathrm{~h}$. After renaturation, the proteins were eluted 
with $0.3 \mathrm{M}$ imidazole in the refolding buffer. The eluted proteins were first dialyzed to remove the glutathione in 0.1 $\mathrm{M}$ urea and $50 \mathrm{mM}$ Tris- $\mathrm{HCl}(\mathrm{pH} \mathrm{8.0)}$ ), followed by dialysis in $0.05 \mathrm{mM}$ urea and $50 \mathrm{mM}$ Tris- $\mathrm{HCl}(\mathrm{pH} 8.0)$. Protein concentration was determined as previously described (Bradford, 1976).

\section{Determination of the activity of the expression product by MTT}

Lymphocytes were isolated from the blood of Xinjiang Wild Argali using the separation medium (TBD, Ltd, Tianjing, China) after incubation for $24 \mathrm{~h}$. The cells were inoculated on the 96-well plates at $1 \times 10^{5}$ cells/well, 20, 40 and $80 \mu \mathrm{g} / \mathrm{mL}$ purified products were added, respectively. Meanwhile, another two 96-well plates were set as the control group, (ConA was added as the negative control (Solarbio Biotechnology, Ltd, Shanghai), while nothing was added the blank control). The three 96 -well plates were in $37^{\circ} \mathrm{C}, 5 \% \mathrm{CO}_{2}$ for $24 \mathrm{~h}$, then $5 \mathrm{mg} / \mathrm{mL}$ MTT (Amresco company, Shanghai, China) was added in each well for another $4 \mathrm{~h}$. Supernatant was removed and the precipitated formazan was dissolved with $100 \mu \mathrm{L}$ dimethyl sulfoxide (Wolsen company, Xi'an, China) followed by gentle shaking of the dishes. Optical density of the dissolved formazan was measured at $570 \mathrm{~nm}$ by using Thermo Labsystems (Roche, Shanghai, China). Finally, statistical analysis was carried out for the data by using one-way ANOVA by SPPSS13.0.

\section{RESULTS}

\section{RACE and complete ISG15 CDNA PCR products}

Nucleotide sequencing combined with a search of the GenBank database revealed five clones generated by 5'RACE-PCR ( $\approx 500$ ) (Figure 1), as having no the initiator element in their open reading frames. The 3 'RACE-PCR products (Figure 2) had complete 3 'ends containing a polyadenylation tail of $12 \mathrm{~A}$ 's at $67 \mathrm{bp}$ downstream of the stop codon TAG. All the ten transformation clones had

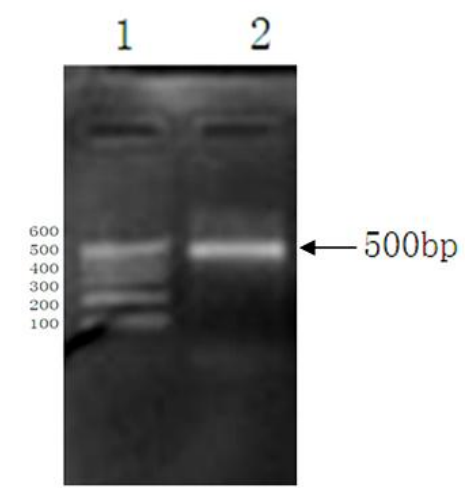

Figure 1. The 5-RACE-PCR products. Lane 1, DNA maker I molecular marker; Lane 2 , positive 3 'ends PCR products $\approx 500 \mathrm{bp}$.

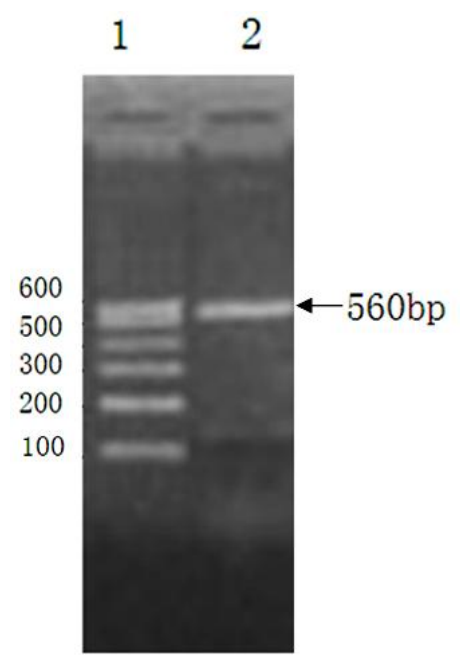

Figure 2. The 3-RACE-PCR products. Lane 1, DNA maker I molecular marker; Lane 2, positive 5'ends PCR product $\approx 560 \mathrm{bp}$.

inserts of the expected ISG15 cDNA-ORF as identified by EcoRI and HindIII enzyme digestion (Figure 3). The sequences were confirmed in all the ten clones. The 3', 5'RACE and full length sequences were 560 bp (3'ends), 500 bp (5'ends) and 642 bp (ISG15 cDNA) respectively (data not shown), each having $99 \%$ identity to Ovis aries mRNA.

\section{Characteristics of ISG15 cDNA sequence}

The sequence of Wild Argali ISG15 cDNA and the inferred amino acids (Figure 4) were submitted to GenBank as the accession no. KC481400. The accuracy of the sequence was determined on both strands and found to be in order. The cDNA insert was 642 bp long. ISG15 cDNAORF contains one long open reading frame of $474 \mathrm{bp}$ from $90 \mathrm{nt}$ to $563 \mathrm{nt}$. The first methionine codon ATG begins at 90-92 nt and ends at presumed stop codon TAG at 561-563

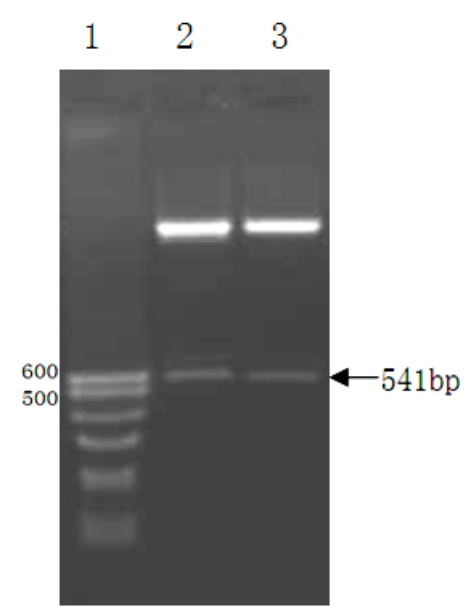

Figure 3. The recombinant plasmids of five clones containing inserts of the full-length ISG15cDNA-ORF identified by EcoRI and HindIII enzyme digestion. Lane 1, DNA maker I molecular marker; Lane $2-3, \approx 541$ bp ISG15-ORF. 


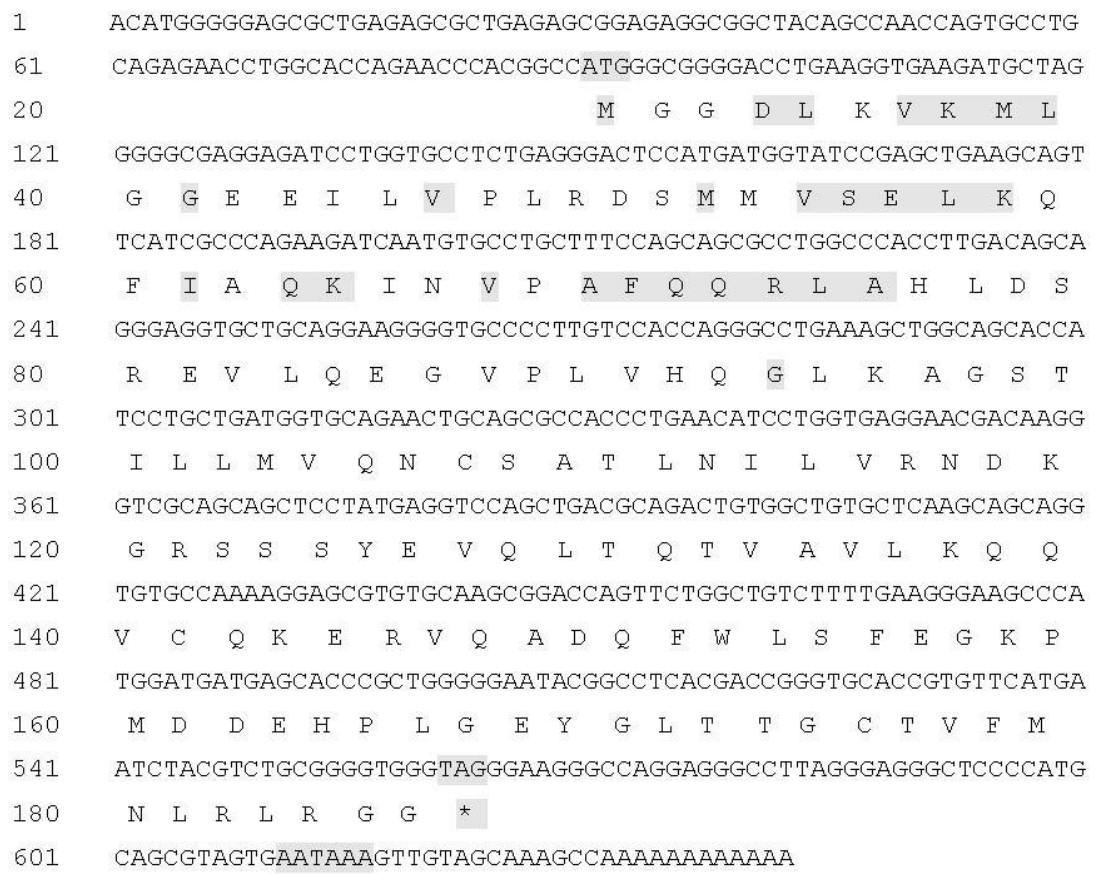

Figure 4. Nucleotide and predicted amino acid sequence of ISG15cDNA. The complete ISG15cDNA sequence consisting of 642 nucleotides and open reading frame of $474 \mathrm{bp}$. The initiation ATG and stop TAG codons are shaded. The distal polyadenylation signal sequence AATAAA are shaded. The nucleotide and its amino acids are numbered along the left margins respectively. Conserved residues of the sequence at G, V, M, I, DL, QK, VKML, VSELK, AFQQRLA are shaded.

nt. The bases AATAAA at 611-626 nt downstream are the distal polyadenylation stop signals for ISG15 cDNA. However, the TATA box was not found in the upstream of ISG15 cDNA -ORF.

The initiation ATG and stop TAG codons are shaded. The distal polyadenylation signal sequence AATAAA are shaded. The nucleotide and its amino acids are numbered along the left margins respectively. Conserved residues of the sequence at G, V, M, I, DL, QK, VKML, VSELK, AFQQRLA are shaded.

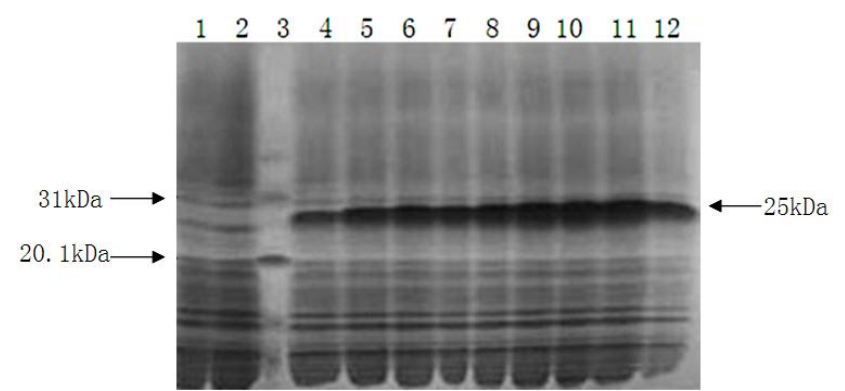

Figure 5. Expression of ISG15 protein. Gels were stained with Coomassie blue and $10 \mu \mathrm{L}$ samples were loaded perlane. Lane 1, pET-28a empty expression vector (negative control); Lane 2, E. coli (BL21 strain) extracts transformed into pET-28a, before induction with IPTG; Lane 3: standard protein molecular weight marker; Lanes 4, 5, 6, 7, 8, 9, 10, 11, and 12, transformed E. coli (BL 21strain) extract by pET-28a, after 0.5, 1, 1.5, 2, 2.5, 3, 4, 5, 6 $\mathrm{h}$ of induction with IPTG, respectively.

\section{Predicted amino acid sequence of recombinant ISG15 cDNA}

The open reading frame encoded a 157 amino acid residue protein with an isoelectric point $(\mathrm{pI})$ of 7.242 and a predicted molecular mass of $17,471.33 \mathrm{Da}(\approx 17.47 \mathrm{kDa})$. The recombinant protein corresponded to the expected molecular mass of $25 \mathrm{kDa}$ as judged by SDS-PAGE and the expression product was detected in the bacterial inclusion bodies (Figures 5 and 6). The protein consists of 54 hydrophobic amino acids (Ala, Ile, Leu, Phe, Trp, and Val), 40 Polar amino acids (N, C, Q, S, T, Y) (Asn, Cys, Gln, Ser, Thr, and Tyr), 17 strongly basic amino acids (Lys and Arg) and 17 strongly acidic amino acids (Asp and Glu). Alignment of amino acid sequences of ISG15 cDNA against

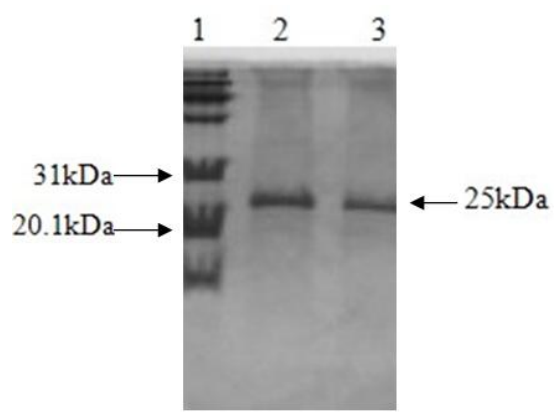

Figure 6. Purification of ISG15 protein. Gels were stained with Coomassie blue and $10 \mu \mathrm{L}$ samples were loaded per lane. Lane 1, standard protein molecular weight marker; Lanes 2 and 3, purified recombinant $25 \mathrm{kDa}$, ISG15 protein. 


\begin{tabular}{|c|c|c|c|c|c|c|c|c|c|c|c|c|}
\hline \multicolumn{13}{|c|}{ Percent Identity } \\
\hline & 1 & 2 & 3 & 4 & 5 & 6 & 7 & 8 & 9 & 10 & & \multirow{3}{*}{ Sus } \\
\hline 1 & & 78.3 & 75.6 & 77.4 & 77.6 & 26.3 & 81.2 & 72.5 & 78.1 & 67.9 & 1 & \\
\hline 2 & 25.9 & & 91.4 & 94.3 & 80.8 & 43.1 & 79.7 & 61.7 & 97.9 & 58.6 & 2 & \\
\hline 3 & 28.5 & 5.0 & & 96.0 & 75.3 & 42.2 & 77.0 & 62.7 & 93.0 & 59.3 & 3 & Bos \\
\hline 4 & 28.5 & 5.2 & 3.0 & & 86.3 & 38.9 & 77.8 & 67.6 & 94.3 & 63.7 & 4 & Bubalus \\
\hline 5 & 27.4 & 10.4 & 12.4 & 9.0 & & 26.8 & 79.1 & 49.5 & 84.4 & 53.5 & 5 & Capra \\
\hline 6 & 89.9 & 89.3 & 90.9 & 80.6 & 90.6 & & 57.0 & 16.8 & 45.1 & 18.7 & 6 & Danio \\
\hline 7 & 20.6 & 25.6 & 28.3 & 27.0 & 26.2 & 77.7 & & 75.5 & 79.5 & 74.7 & 7 & Felis \\
\hline 8 & 33.5 & 45.5 & 47.7 & 39.7 & 47.5 & 105.2 & 29.2 & & 64.7 & 78.0 & 8 & Mus \\
\hline 9 & 26.2 & 1.3 & 4.8 & 5.2 & 9.0 & 85.6 & 25.9 & 41.8 & & 64.5 & 9 & Ovis \\
\hline \multirow[t]{2}{*}{10} & 36.1 & 40.8 & 41.5 & 39.9 & 47.0 & 104.7 & 29.8 & 20.2 & 39.5 & & 10 & Rattus \\
\hline & 1 & 2 & 3 & 4 & 5 & 6 & 7 & 8 & 9 & 10 & & \\
\hline
\end{tabular}

Figure 7. Computer genetic distance from ISG15cDNA sequence. Sequences were derived from Gen bank databases.

amino acid sequences from other species revealed ISG15 protein shared $97.9 \%, 80.8 \%, 91.4 \%, 94.3 \%, 78.3 \%$ identity with those of ISG15 cDNA from Ovis aries (accession no. NM001009735.1), Capra hircus (accession no. HQ329186.1), Bos taurus (accession no. BC102318.1), Bubalus bubalis (accession no. HM543269.1), and Sus scrofa (accession no. EU647216.1), respectively (Figure 7). The phylogenetic tree generated from nucleotide sequences (Figure 8) revealed a close relationship between Argali and Ovis aries.

\section{Effects of recombination protein on lymphocyte proliferation}

The results from lymphocyte proliferation test showed that the expression product could stimulate lymphocyte proliferation very well $(\mathrm{p}<0.05)$, which proved the expressed protein had biological activity (Table 1).

\section{DISCUSSION}

The ISG15 cDNA sequence of the Xinjiang Wild Argali for testing was obtained by RT-PCR, 5'RACE, and 3'RACE methods. The ISG15 cDNA was 642 bp long with an open reading frame of $474 \mathrm{bp}$, which encoded a $17.47 \mathrm{kDa}$ protein composed of 157 amino acids, containing one initiation codon ATG and one termination codon TAG. The
Table 1. Effects of Argali ISG15 protein on lymphocyte proliferation $(\mathrm{X} \pm \mathrm{S}),(\mathrm{p}<0.05)$

\begin{tabular}{ll}
\hline Concentration $(\mu \mathrm{g} / \mathrm{mL})$ & $\mathrm{OD}_{570 \mathrm{~nm}}$ \\
\hline 40 (ConA) & $0.1770 \pm 0.0102^{\mathrm{ab}}$ \\
0 (Control) & $0.1067 \pm 0.0034^{\mathrm{a}}$ \\
20 (ISG15) & $0.1410 \pm 0.0057^{\mathrm{ab}}$ \\
40 (ISG15) & $0.1763 \pm 0.0061^{\mathrm{b}}$ \\
80 (ISG15) & $0.1980 \pm 0.0245^{\mathrm{b}}$ \\
\hline
\end{tabular}

5'UTR ends at $89 \mathrm{nt}$ and the 3'UTR ends at 79 bp in Argali, while the 5'UTR ends at $91 \mathrm{nt}$, the 3'UTR ends at $81 \mathrm{bp}$ in sheep. We found that Wild Argali, goats and sheep showed the highest homology by BLAST, which was in accordance with our expectation. The bases AATAAA at 611-626 nt downstream are the distal polyadenylation stop signals for ISG15 cDNA. However, the TATA box was not found in the upstream of ISG15 cDNA-ORF. Song reported the results for ISG15 cDNA cloning from small tail cold sheep and jining grey goats where the 3' UTR retained CdxA binding sites for transcription even in the case of frame shift, CdxA components were generally located in the 5' end of the gene and worked as a part of the promoter (Song, 2007). All of these results suggest that the $\mathrm{CdxA}$ transcription factor may regulate the expression of $I S G 15$ gene. The difference in the non-coding regions of $I S G 15$ gene between wild argali and

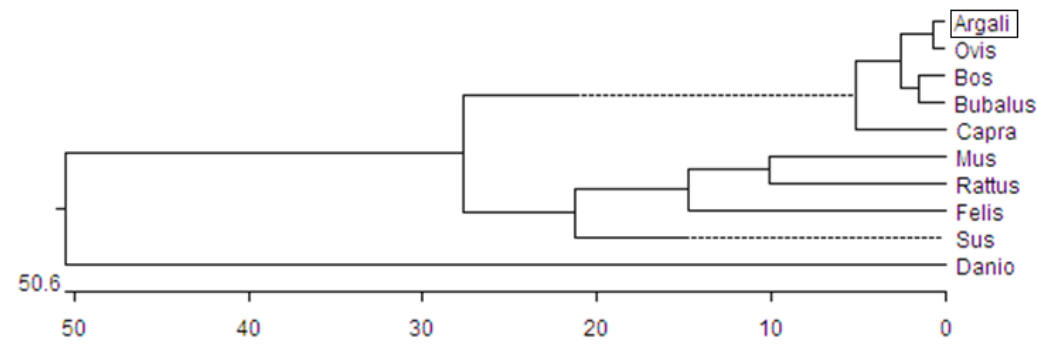

Figure 8. Computer generated phylogenetic tree from ISG15cDNA sequences. Sequences were derived from Gen bank databases. 
sheep suggests that there may be differences in the regulation of ISG15 expression by CdxA transcription factor among different species.

$I S G 15$, as a disease resistance related gene, plays an important role in regulating innate immunity (Okumura et al., 2006). Ritchie et al. confirmed that ISG15 and covalent modifications in ISG15 played important roles in stimulating lymphocyte proliferation, enhancing cellular antiviral action and promoting leukocyte chemotaxis (Ritchie et al., 2004). Moreover, ISG15 is able to induce IFN- $\gamma$ of $\mathrm{T}$ cell expression, stimulate proliferation of natural killer cells, and improve its cytotoxic effect (Kim et al., 2003). ISG15 can regulate immune cell activation and proliferation (Pitha-Rowe et al., 2007). Therefore, MTT method was used to detect the activity of the recombinant ISG15 protein in this study.

The results showed that the recombinant ISG15 protein can stimulate lymphocyte proliferation and had certain biological activity. Previously, in order to make full use of xinjiang wild argali's superior performance and the characteristics of high disease resistance, argali were crossed with Xinjiang local sheep. The hybrids grew fast and had a larger and better production performance. However, it was found that hybrids suffered from poor disease resistance, they were susceptible to respiratory disease, and the mortality was high during early growth and development.

Cui found after comparing the ISG15 gene sequences of parents' generation and hybrids that there were differences among them (Cui, 2012). Therefore, the present study was carried out to clone and express the ISG15 gene of the argali, which will provide a basis for further studies on the differences in gene functions and disease resistance.

\section{ACKNOWLEDGEMENTS}

The present study was funded by a grant from the National Natural Science Foundation of P. R. China (No. 31060351).

\section{REFERENCES}

Bradford, M M. 1976. A rapid and sensitive method for the quantitation of microgram quantities of protein utilizing the principle of protein-dye binding. Anal. Biochem. 72:248-254.
Chang, L., Q. Wen-Tao, W. Chen, and G. Yun-Qi. 2006. Ubiquitinlike protein ISG15 and its role in innate immunity. Prog. Biochem. Biophys. 33:1023-1029.

Cui, R. P. 2012. Cloning, expression and activity detection of ubiquitin ISG15 of Argal, Ms. Barsh Worship and Hybrid Sheep, Shihezi University.

Der, S. D., A. Zhou, B. R. Williams, and R. H. Silverman. 1998. Identification of genes differentially regulated by interferon $\alpha$, $\beta$, or $\gamma$ using oligonucleotide arrays. Proc. Natl. Acad. Sci. USA 95:15623-15628.

Jueken, A., J. Kumunisihan, H. Hamiti, Y. Sulaiman, X. S. Yu, M. Du, Hailati, Tuersenhali, and Ayinuer. 2007. Study on hybridication of wild Argali and Bashibai sheep. Xinjiang Agric. Sci. 5:702-705.

Kim, K. I. and D. E. Zhang. 2003. ISG15, not just another ubiquitin-like protein. Biochem. Biophys. Res. Commun. 307: 431-434.

Okumura, A., G. Lu, I. Pitha-Rowe, and P. M. Pitha. 2006. Innate antiviral response targets HIV-1 release by the induction of ubiquitin-like protein ISG15. Proc. Natl. Acad. Sci. USA 103: 1440-1445.

Pitha-Rowe, I. F. and P. M. Pitha. 2007. Viral defense, carcinogenesis and ISG15: novel roles for an old ISG. Cytokine Growth Factor Rev. 18:409-417.

Rempel, L. A., B. R. Francis, K. J. Austin, and T. R. Hansen. 2005. Isolation and sequence of an interferon- $\tau$-inducible, pregnancy-and bovine interferon-stimulated gene product 15 (ISG15)-specific, bovine ubiquitin-activating E1-like (UBE1L) enzyme. Biol. Reprod. 72:365-372.

Ritchie, K. J., C. S. Hahn, K. I. Kim, M. Yan, D. Rosario, L. Li, J. C. Torre, and D. E. Zhang. 2004. Role of ISG15 protease UBP43 (USP18) in innate immunity to viral infection. Nat. Med. 10:1374-1378.

Song, X. M. 2007. Small-tail han sheep reproduction and disease resistance relational genes cloning and comparative genomics analysis. Institute of Animal Sciences, Chinese Academy of Agricultural Sciences, 6.

Yu, Y., M. Ji,C. Liu, and K. Li. 2008. Songtao Guo Northwest Institute of Endangered Animal Species, Xi'an 710032; Geographical distribution and vicissitude of argali, Ovis ammon. China, 2.

Zhang, D. and D. E. Zhang. 2011. Interferon-stimulated gene 15 and the protein ISGylation system. J. Interferon Cytokine Res. 31:119-130.

Zhao, C., S. L. Beaudenon, M. L. Kelley, M. B. Waddell, W. Yuan, B. A. Schulman, J. M. Huibregtse, and R. M. Krug. 2004. The UbcH8 ubiquitin E2 enzyme is also the E2 enzyme for ISG15, an IFN-alpha/beta-induced ubiquitin-like protein. Proc. Natl. Acad. Sci. USA 101:7578-7582. 